\title{
The pharmacology of multiple regimens of agalsidase alfa enzyme replacement therapy for Fabry disease
}

Joe T. R. Clarke, $M D, P h D^{1,2}$ Michael L. West, $M D^{3}$, Jan Bultas, $M D, P h D^{4}$, and Raphael Schiffmann, $M D^{5}$

\begin{abstract}
Purpose: This 10-week study was conducted to determine the pharmacokinetics of varying doses of agalsidase alfa and evaluate the effect of dose and dosing frequency on plasma $\mathrm{Gb}_{3}$ levels. Methods: Eighteen adult male Fabry patients, naive to enzyme replacement therapy, were randomized to one of five regimens: $0.1,0.2$, or $0.4 \mathrm{mg} / \mathrm{kg}$ weekly; $0.2 \mathrm{mg} / \mathrm{kg}$ every other week (the approved dose); or $0.4 \mathrm{mg} / \mathrm{kg}$ every other week. Intravenous infusion rate was $0.1 \mathrm{mg} / \mathrm{kg}$ per 20 minutes. Plasma $\mathrm{Gb}_{3}$ levels were assessed at baseline and periodically during the study. Results: The mean half-life was 56-76 minutes, and the mean volume of distribution at steady state was $17 \%-18 \%$ of body weight, with no significant association between dose and half-life, clearance, or volume of distribution at steady state. The area under the curve was linearly proportional to the dose from 0.1 to $0.4 \mathrm{mg} / \mathrm{kg}$. Baseline average plasma $\mathrm{Gb}_{3}$ was $9.12 \pm 2.61 \mathrm{nmol} / \mathrm{mL}$ and after 10 weeks of treatment was significantly reduced by about $50 \%$ in each group with no statistically significant differences between groups. Conclusions: Reduction of plasma $\mathrm{Gb}_{3}$ levels was independent of dose or dose frequency in the range tested. These observations, coupled with the clinical trial experience of both agalsidase alfa and agalsidase beta, indicate that the standard dose of agalsidase alfa is sufficient to maximally reduce plasma $\mathrm{Gb}_{3}$. However, because plasma $\mathrm{Gb}_{3}$ is not a validated surrogate of disease severity in Fabry disease, further clinical study will be required to determine the optimal dosing regimen for providing maximal clinical benefit. Genet Med 2007:9(8):504-509.
\end{abstract}

Key Words: Fabry disease, agalsidase alfa, enzyme replacement therapy, pharmacodynamics, dose response

Fabry disease is an X-linked inherited metabolic disorder caused by deficiency of the activity of the lysosomal enzyme, $\alpha$-galactosidase A ( $\alpha$-Gal A). ${ }^{1}$ It occurs with an estimated incidence of about 0.85 per $100,000,{ }^{2}$ although a recent study suggests a much higher incidence. ${ }^{3}$ In affected subjects, the principal substrate of the enzyme, globotriaosylceramide

From the ${ }^{1}$ Division of Clinical and Metabolic Genetics, The Hospital for Sick Children and University of Toronto, Toronto, Ontario, Canada; ${ }^{2}$ Service de génétique médicale, Centre hospitalier universitaire de Sherbrooke, Sherbrooke, Quebec, Canada; ${ }^{3}$ Dalhousie University, Halifax, Nova Scotia, Canada; ${ }^{4}$ Department of Pharmacology, $3^{\text {rd }}$ Faculty of Medicine, Charles University Hospital, Prague, Czech Republic; ${ }^{5}$ National Institute of Neurological Disease and Stroke, National Institutes of Health, Bethesda, Maryland.

Disclosure: Dr. Clarke has received research grants, unrestricted educational grants, and honoraria for scientific and continuing medical education presentations regarding the management of lysosomal storage diseases from Genzyme Corporation, Genzyme Canada, Shire Human Genetic Therapies, and Actelion Pharmaceuticals Canada. He also receives honoraria for service on advisory boards sponsored by the same companies regarding the management of lysosomal storage diseases., Dr. West has received research funds, has been a consultant to and received honoraria from both Genzyme Corporation and Shire Pharmaceuticals. Drs. Bultas and Schiffmann have no conflicts of interest.

Joe T.R. Clarke, MD, PhD, Division of Clinical and Metabolic Genetics The Hospital for Sick Children, 555 University Avenue, Toronto, Ontario M5G 1X8, Canada; e-mail: jtrc@sickkids.ca

Submitted for publication April 20, 2007.

Accepted for publication May 31, 2007.

DOI: 10.1097/GIM.0b013e318133fb1b
$\left(\mathrm{Gb}_{3}\right)$, accumulates within tissues and organs, including skin, heart, all cells of the kidney, vascular endothelial and smooth muscle cells, eye, and cells of the autonomic nervous system, ${ }^{4,5}$ resulting in progressive tissue damage and associated clinical problems. The onset of signs and symptoms of Fabry disease in affected males is generally in childhood and adolescence ${ }^{6-8}$ usually beginning with neuropathic pain. Other signs and symptoms of Fabry disease include poor sweating, ${ }^{9-11}$ hearing loss, ${ }^{12}$ hypertrophic cardiomyopathy, ${ }^{13-15}$ valvular heart disease, ${ }^{15}$ cardiac conduction abnormalities, ${ }^{16}$ progressive kidney dysfunction, ${ }^{6-9,17,18}$ a high incidence of stroke and transient ischemic attacks, ${ }^{9}, 10$ and impaired quality of life. ${ }^{19,20}$ The median age at death of male Fabry disease patients has been reported to be 50 years of age, with renal failure, stroke, and cardiovascular disease as major contributors. ${ }^{9}$ Although the disease is X-linked, signs and symptoms are often reported by female heterozygotes, with the onset delayed by about 10 years and the severity and progression more variable than in the male Fabry population..$^{10,21}$

Before the availability of enzyme replacement therapy (ERT), management of Fabry disease was limited to symptomatic and supportive therapy. However, the introduction of recombinant human $\alpha$-Gal A for ERT now makes treatment directed at the basic biochemical defect of Fabry disease possible. Two formulations of recombinant $\alpha$-Gal A are currently avail- 
able for ERT of Fabry disease: agalsidase alfa and agalsidase beta. Although the two enzymes share the same amino acid sequence as the endogenous $\alpha$-Gal A, the glycosylation patterns are different, ${ }^{22}$ primarily because of their different manufacturing processes. Agalsidase alfa (Replagal; Shire Human Genetic Therapies, Inc., Cambridge, MA) is produced in a cultured human cell line ${ }^{23}$ and is approved (agalsidase alfa is not currently approved in the United States) for use at a dose of 0.2 $\mathrm{mg} / \mathrm{kg}$, infused intravenously every other week (EOW). Agalsidase beta (Fabrazyme, Genzyme Corporation, Cambridge, MA) is produced by cultured Chinese hamster ovary cells and is recommended for use at a dose of $1.0 \mathrm{mg} / \mathrm{kg}$, infused intravenously EOW. Double-blind, placebo-controlled clinical trials of both enzymes at their approved doses have shown clinical benefit. Agalsidase alfa at $0.2 \mathrm{mg} / \mathrm{kg}$ EOW reduced the severity of neuropathic pain in adult male Fabry disease patients after 6 months of treatment. ${ }^{24}$ In a median 18.5-month trial in Fabry disease patients with evidence of kidney dysfunction, agalsidase beta at $1.0 \mathrm{mg} / \mathrm{kg}$ EOW slowed the progression to the primary endpoint, a composite of renal, cardiac, and cerebrovascular events. ${ }^{25}$ In vitro testing has shown that the enzymatic activity of these two preparations is indistinguishable, prompting some to suggest that the approved dose of agalsidase alfa $(0.2 \mathrm{mg} / \mathrm{kg}$ EOW $)$ may be insufficient for maximal clinical benefit. ${ }^{22}$

If agalsidase alfa at $0.2 \mathrm{mg} / \mathrm{kg}$ EOW is insufficient for producing maximal clinical benefit, increasing the dose or the frequency of administration should increase its efficacy. For example, the tissue half-life of agalsidase alfa is estimated to be between 24 and 48 hours $^{26}$; therefore, little or no enzyme would be present in tissue and organs during the second week after dosing. Thus, switching to weekly dosing might provide more even cellular protection and could result in a larger clinical benefit. Here we describe the results of short-term pharmacokinetic and pharmacodynamic studies designed to evaluate the effect of changing the dose or dose frequency of agalsidase alfa on the plasma $\mathrm{Gb}_{3}$ levels in male Fabry patients.

\section{MATERIALS AND METHODS}

\section{Patients}

Male ERT-naive patients 18 years or older with a confirmed diagnosis of Fabry disease were eligible for inclusion in the study. Fabry disease could be confirmed by mutation analysis or by a demonstration of a deficiency of $\alpha$-Gal A activity $(<4.0$ $\mathrm{nmol} / \mathrm{mL}$ per hour in plasma or serum or $<8 \%$ of the average mean normal in leukocytes). In addition, patients must have had at least one sign or symptom of Fabry disease, including neuropathic pain, angiokeratoma, corneal verticillata, cardiomyopathy, hypo- or anhydrosis, abdominal pain and/or diarrhea, serum creatinine $>1.0 \mathrm{mg} / \mathrm{dL}$, and proteinuria $>300$ $\mathrm{mg} / 24 \mathrm{hr}$. Exclusion criteria included being on kidney dialysis or having received a kidney transplant.

\section{Study design}

The study was an open-label, randomized, multicenter clinical trial designed to investigate the pharmacokinetics and pharmacodynamics of various dosing regimens of agalsidase alfa. The protocol was approved by the institutional review board or an independent ethics committee at each site, and all patients signed informed consent documents before enrolling in the study. Participating study sites included the National Institutes of Health, Bethesda, MD (four patients); Hospital for Sick Children, Toronto, ON, Canada (seven patients); Dalhousie University, Halifax, NS, Canada (three patients), and the Charles University, Prague, Czech Republic (four patients).

Patients were randomly assigned to one of five dosing groups stratified by baseline plasma $\mathrm{Gb}_{3}$ levels (low, 4 to $<11.5$ $\mathrm{nmol} / \mathrm{mL}$; high, $\geq 11.5 \mathrm{nmol} / \mathrm{mL}$ ). The treatment groups were (1) $0.1 \mathrm{mg} / \mathrm{kg}$ weekly, (2) $0.2 \mathrm{mg} / \mathrm{kg}$ EOW (the standard dose), (3) $0.2 \mathrm{mg} / \mathrm{kg}$ weekly, (4) $0.4 \mathrm{mg} / \mathrm{kg}$ EOW, and (5) $0.4 \mathrm{mg} / \mathrm{kg}$ weekly. All doses were infused intravenously at a rate of 0.1 $\mathrm{mg} / \mathrm{kg}$ per 20 minutes. The study was of 10 weeks' duration. Thus, patients treated weekly received 10 infusions and patients treated EOW received five infusions. Informed consent was obtained from all patients, and the study received appropriate institutional review board approval.

\section{Pharmacokinetics}

Pharmacokinetics (PK) studies were performed over 8 hours after the initial infusion of each dose of agalsidase alfa. Blood samples were taken periodically during and after the infusion, with the timing of the samples and duration of sampling determined by the dose. Blood samples were allowed to clot and were centrifuged within 30 minutes, with the resulting serum stored at $-70^{\circ} \mathrm{C}$ until analysis. PK parameters were calculated using a noncompartmental model (WinNonlin Professional version 4.1, Model 202, Pharsight Corporation, Mountain View, CA) and were based on the $\alpha$-Gal A activity in serum. Serum $\alpha$-Gal A activity was determined at a central laboratory (Shire Human Genetic Therapies) and was measured with the use of the synthetic substrate, 4-methylumbelliferyl- $\alpha$-D-galactopyranoside, and an in vitro fluorescence assay. ${ }^{27}$ The following PK parameters were calculated: area under the curve (AUC) extrapolated to infinity (AUC, $\min \bullet U / m L)$, maximum serum concentration $\left(C_{\max }, U / m L\right)$, time of $\mathrm{C}_{\max }\left(\mathrm{T}_{\max }\right.$, in minutes), terminal elimination half-life $\left(t_{1 / 2}\right.$, in minutes), mean residence time (MRT, in minutes), serum clearance rate $(\mathrm{Cl}, \mathrm{mL} / \mathrm{min}), \mathrm{Cl}$ normalized for body weight $(\mathrm{mL} / \mathrm{min} / \mathrm{kg})$, and apparent volume of distribution at steady state $\left(\mathrm{V}_{\mathrm{ss}}\right.$, in liters).

\section{Pharmacodynamic studies}

The primary efficacy endpoint variable was change in plasma $\mathrm{Gb}_{3}$ level from baseline to the day of the final infusion. Plasma $\mathrm{Gb}_{3}$ was also measured weekly during the study and 7 and 30 days after the final infusion. Plasma samples were frozen at $-70^{\circ} \mathrm{C}$ until analysis. Plasma $\mathrm{Gb}_{3}$ levels were deter- 
mined at Shire HGT by an high-performance liquid chromatography method previously described. ${ }^{23}$

\section{Safety}

Before the first infusion, all patients underwent a baseline evaluation that included a physical examination; clinical laboratory tests including chemistry, hematology, coagulation, urinalysis, and 24-hour urine chemistry; and an electrocardiogram. Treatment-associated adverse events and the results of periodic repetition of the above tests were used to evaluate safety. Serum was analyzed for the presence of anti-agalsidase alfa antibodies by enzyme-linked immunosorbent assays. All samples having a time point absorbance $>0.04$ and a time point/baseline absorbance ratio $>2.0$ were considered positive. Positive samples were further titrated to confirm the presence of antibodies.

\section{Statistical analysis}

All statistical analyses were done with SAS software, version 8.2 (Cary, NC). Analysis of variance was used to compare the effects of various dosing regimens on plasma $\mathrm{Gb}_{3}$ or on pharmacokinetic parameters. Other tests are indicated in the text. Values are expressed as mean \pm SD.

\section{RESULTS}

A total of 18 adult male Fabry disease patients were enrolled, and all completed the 10-week study. Their demographic and baseline disease characteristics are shown in Table 1. One patient was younger than 18 years old and was given an exemption to enroll in the study.

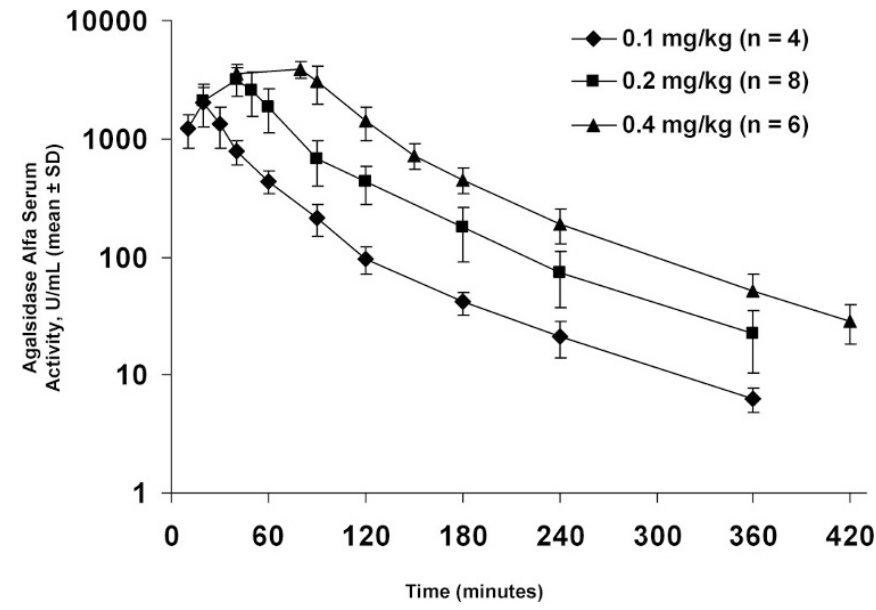

Fig. 1. Serum clearance curves after intravenous infusion of agalsidase alfa at $0.1,0.2$, or $0.4 \mathrm{mg} / \mathrm{kg}$ in male Fabry disease patients. The infusion times were 20,40 , and 80 minutes for the $0.1,0.2$, and $0.4 \mathrm{mg} / \mathrm{kg}$ doses, respectively.

\section{Pharmacokinetics}

The serum clearance curves measured during and after the initial doses of agalsidase alfa are presented in Figure 1, and the calculated pharmacokinetic parameters are shown in Table 2. The peak serum concentration $\left(\mathrm{C}_{\max }\right)$ was proportional to dose and coincided with the end of the 20-, 40-, or 80-minute infusion period (Fig. 1). No significant association of dose was found for $t_{1 / 2}, \mathrm{Cl}$, or $\mathrm{V}_{\mathrm{ss}}$. Mean $t_{1 / 2}$ was approximately $65 \mathrm{~min}$ utes (range for individual patients, 56-76 minutes). The mean volume of distribution at steady state $\left(\mathrm{V}_{\mathrm{ss}}\right)$ was $17 \%-18 \%$ of body weight. The AUC was linearly proportional to dose from 0.1 to $0.4 \mathrm{mg} / \mathrm{kg}$ (regression analysis indicated $R^{2}=0.996, P<$ 0.001 ), indicating that serum clearance mechanisms had not

Table 1

Demographic and baseline Fabry disease characteristics

\begin{tabular}{|c|c|c|c|c|c|}
\hline & \multicolumn{5}{|c|}{ Treatment group } \\
\hline & $0.1 \mathrm{mg} / \mathrm{kg}$ weekly & $0.2 \mathrm{mg} / \mathrm{kg}$ EOW & $0.2 \mathrm{mg} / \mathrm{kg}$ weekly & $0.4 \mathrm{mg} / \mathrm{kg}$ EOW & $0.4 \mathrm{mg} / \mathrm{kg}$ weekly \\
\hline No. & 4 & 4 & 4 & 3 & 3 \\
\hline Age, yr, mean (range) & $27.5(16-36)$ & $37.5(33-41)$ & $25.0(20-32)$ & $23.0(21-25)$ & $28.3(26-30)$ \\
\hline $\begin{array}{l}\text { Time since diagnosis, yr, } \\
\text { mean (range) }\end{array}$ & $6.9(0.08-20)$ & $17.4(0.5-24)$ & $14.4(3.5-20)$ & $6.0(0.08-14)$ & $5.5(1.5-12)$ \\
\hline Race (white/Hispanic) & $4 / 0$ & $4 / 0$ & $4 / 0$ & $1 / 2$ & $3 / 0$ \\
\hline Neuropathic pain, no. & 4 & 4 & 4 & 3 & 3 \\
\hline Abdominal pain, no. & 4 & 2 & 3 & 3 & 0 \\
\hline Diarrhea, no. & 3 & 3 & 2 & 3 & 2 \\
\hline Ear signs and symptoms, no. & 1 & 2 & 2 & 0 & 0 \\
\hline Corneal verticillata, no. & 2 & 3 & 2 & 0 & 1 \\
\hline Renal dysfunction, ${ }^{a}$ no. & 2 & 4 & 1 & 1 & 1 \\
\hline $\begin{array}{l}\text { Baseline plasma } \mathrm{Gb}_{3} \text {, } \\
\mathrm{nmol} / \mathrm{mL} \text {, mean (range) }\end{array}$ & $9.82(7.3-13.0)$ & $9.72(4.8-4.4)$ & $8.95(6.3-11.2)$ & $7.76(5.3-9.9)$ & $8.96(7.3-11.4)$ \\
\hline
\end{tabular}

EOW, every other week; $\mathrm{Gb}_{3}$, globotriaosylceramide; no., number of patients.

${ }^{a}$ Abnormal serum creatinine and/or proteinuria $>300 \mathrm{mg} / 24 \mathrm{hr}$. 
Table 2

Pharmacokinetic parameters from the initial dose of agalsidase alfa in male Fabry disease patients

\begin{tabular}{lccccc}
\hline $\begin{array}{l}\text { Dose group } \\
(\mathrm{mg} / \mathrm{kg})\end{array}$ & No. & $\begin{array}{c}\mathrm{C}_{\max } \\
\left(\mathrm{U} / \mathrm{kg} \bullet 10^{6}\right)\end{array}$ & $\begin{array}{c}\mathrm{AUC} \\
(\mathrm{min} \bullet \mathrm{U} / \mathrm{mL})\end{array}$ & $\begin{array}{c}\mathrm{Cl} \\
(\mathrm{mL} / \mathrm{min} / \mathrm{kg})\end{array}$ & $\begin{array}{c}t_{1 / 2} \\
(\mathrm{~min})\end{array}$ \\
\hline 0.1 & 4 & $2046 \pm 672$ & $84321 \pm 23995$ & $4.02 \pm 1.03$ & $66 \pm 3$ \\
0.2 & 8 & $3148 \pm 849$ & $212296 \pm 74550$ & $3.43 \pm 1.47$ & $6.173 \pm 0.049$ \\
0.4 & 6 & $4168 \pm 418$ & $413698 \pm 72559$ & $3.17 \pm 0.52$ & $65 \pm 4$ \\
\hline
\end{tabular}

All values mean $\pm \mathrm{SD}$. $\left(\mathrm{C}_{\max }\right.$, maximum serum concentration $\mathrm{AUC}$, area under the curve; $\mathrm{Cl}$, serum clearance rate; $t_{1 / 2}$ terminal elimination half-life; $\mathrm{V}_{\mathrm{ss}}$, apparent volume of distribution at steady state.

become saturated even at the highest dose tested. Consistent with this observation, mean $\mathrm{Cl}$ values (normalized for body weight: $4.02,3.43,3.17 \mathrm{~mL} / \mathrm{min} / \mathrm{kg}$ for $0.1,0.2$, and $0.4 \mathrm{mg} / \mathrm{kg}$ doses, respectively), were not statistically significantly different (analysis of variance, $P=0.53$ ).

\section{Change in plasma $\mathbf{G b}_{3}$}

At baseline, the average plasma $\mathrm{Gb}_{3}$ was $9.12 \pm 2.61$ $\mathrm{nmol} / \mathrm{mL}$ (range, $4.8-14.4 \mathrm{nmol} / \mathrm{mL}$ ). The average plasma $\mathrm{Gb}_{3}$ level was reduced by all dosing regimens (Fig. 2). After 10 weeks of treatment, the average plasma $\mathrm{Gb}_{3}$ was decreased by about $50 \%$ for each dosing regimen (Fig. 3), reaching an overall average of $4.65 \pm 1.62 \mathrm{nmol} / \mathrm{mL}$ (range, $3.2-9.5 \mathrm{nmol} / \mathrm{mL}$ ). For the entire pooled patient population, the average plasma $\mathrm{Gb}_{3}$ was $51.5 \% \pm 14.3 \%$ of the baseline value $(P<0.001)$. No statistically significant association between the magnitude of the reduction in plasma $\mathrm{Gb}_{3}$ and dose or dose frequency was found (analysis of variance). Interestingly, average plasma $\mathrm{Gb}_{3}$ levels remained reduced for at least 4 weeks after the final dose.

\section{Safety}

The majority of patients experienced one or more treatment-associated adverse events. These events were all typical manifestations or complications of Fabry disease or were problems commonly reported in the general population. The incidence of adverse events was not related to dose or dosing fre-

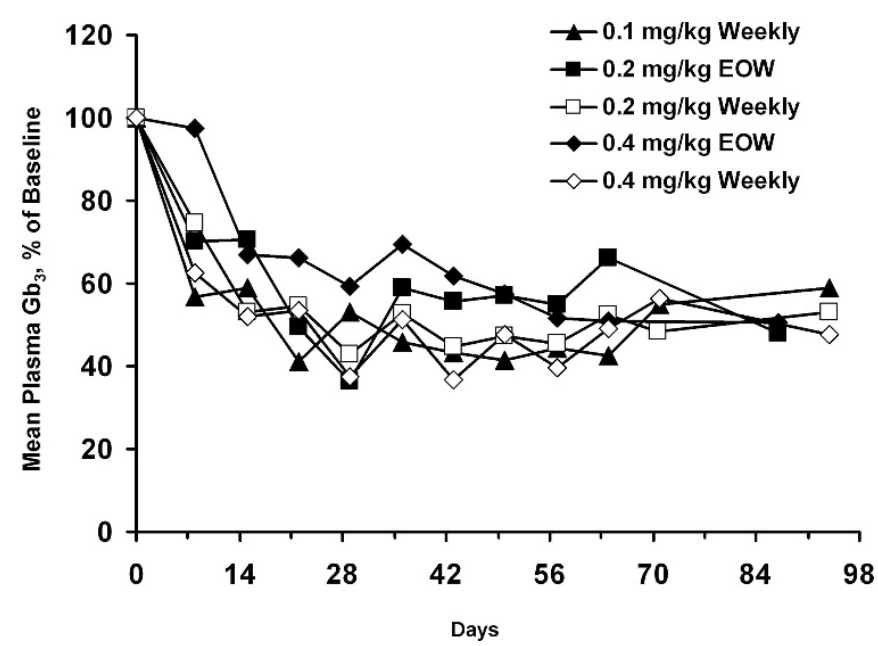

Fig. 2. Effect of five different dosing regimens of agalsidase alfa on mean plasma globotriaosylceramide $\left(\mathrm{Gb}_{3}\right)$ level in male Fabry disease patients. EOW, every other week. quency. Infusion reactions, which typically consisted of pyrexia and/or rigors, occurred in five of the 18 patients (28\%), including three patients in the $0.1-\mathrm{mg} / \mathrm{kg}$ weekly group (one of whom had seven mild infusion reactions) and two patients in the $0.4-\mathrm{mg} / \mathrm{kg}$ EOW group (one of whom had three mild infusion reactions). Only one infusion was interrupted and restarted because of an infusion reaction, and no patient discontinued therapy because of an infusion reaction. Two of 18 of patients $(11 \%)$, both in the $0.1-\mathrm{mg} / \mathrm{kg}$ weekly group, became antiagalsidase alfa IgG positive during the study. No IgE antiagalsidase alfa antibodies were detected during the study.

\section{DISCUSSION}

In this study, five different dosing regimens of agalsidase alfa were evaluated in male Fabry patients not previously exposed to ERT. Each dosing regimen reduced plasma $\mathrm{Gb}_{3}$ by about $50 \%$. Thus, the currently recommended dosing regimen, 0.2 $\mathrm{mg} / \mathrm{kg}$ EOW, was as pharmacodynamically effective as the higher doses or the more frequent doses. No dose-related safety issues were apparent during this study. The results of the pharmacokinetic studies, which revealed no dose-related influences on plasma half-life, $\mathrm{V}_{\mathrm{ss}}$, or AUC, indicated that the effectiveness of agalsidase alfa treatment on plasma $\mathrm{Gb}_{3}$ levels at the higher doses is not likely limited by some saturable uptake mechanism.

As noted above, two forms of human $\alpha$-Gal A are available for ERT in Fabry disease, and each is approved at a different dose. The original dosing regimen for agalsidase alfa $(0.2$ $\mathrm{mg} / \mathrm{kg}$ EOW) was selected based on calculations of the meta-

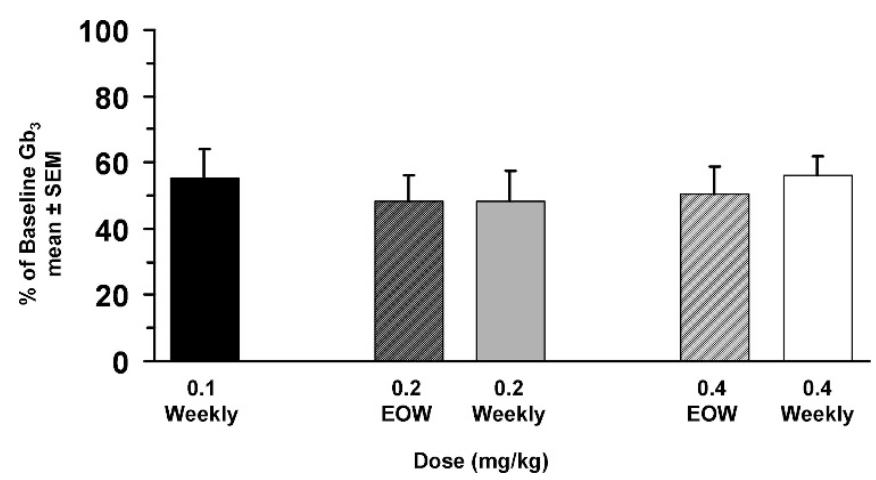

Fig. 3. Plasma globotriaosylceramide $\left(\mathrm{Gb}_{3}\right)$ levels after 10 weeks of dosing in male Fabry disease patients. 
bolic load of $\mathrm{Gb}_{3}$ produced systemically combined with the observation that liver $\mathrm{Gb}_{3}$ and urine sediment $\mathrm{Gb}_{3}$ could be reduced with a single infusion of agalsidase alfa..$^{23}$ In addition, the use of EOW dosing was supported by the positive experience with EOW ERT in Gaucher disease. ${ }^{28}$ In the case of agalsidase beta, the dose of $1 \mathrm{mg} / \mathrm{kg}$ EOW appears to have been chosen based on the results of a dose-response study that showed a rapid reduction of plasma $\mathrm{Gb}_{3}$ levels to near normal at this dose, coupled with a substantial reduction in $\mathrm{Gb}_{3}$ content of liver and vascular endothelial cells. ${ }^{29}$

In the pivotal clinical studies of each of these forms of $\alpha$-Gal $\mathrm{A}$, the magnitude of the reduction in plasma $\mathrm{Gb}_{3}$ appeared to be different. With agalsidase beta, plasma $\mathrm{Gb}_{3}$ levels were reported to be reduced by $100 \%$ with $1 \mathrm{mg} / \mathrm{kg}$ EOW. ${ }^{30} \mathrm{In}$ contrast, agalsidase alfa reduced plasma $\mathrm{Gb}_{3}$ levels by about $54 \%$ with $0.2-\mathrm{mg} / \mathrm{kg}$ EOW dosing. ${ }^{24}$ This apparent dose response had led some to speculate that agalsidase alfa was being used at a suboptimal dose ${ }^{31}$; however, the results of the long-term open-label extension study of agalsidase beta showed sustained reductions in plasma $\mathrm{Gb}_{3}$ levels of only $50 \%$ after 18 or more months of treatment at $1 \mathrm{mg} / \mathrm{kg}$ EOW. ${ }^{32}$ This apparent change in potency was likely an artifact due to a change from an enzyme-linked immunosorbent assay method to a more sensitive mass spectrometry for assaying plasma $\mathrm{Gb}_{3} \cdot{ }^{32,33}$

In the current study, changes in plasma $\mathrm{Gb}_{3}$ were used as a biomarker for the evaluation of the response to various doses and dosing regimens of agalsidase alfa. Combined with clinical trial experience, ${ }^{24,34}$ the results show that the maximum reduction in plasma $\mathrm{Gb}_{3}$ with agalsidase alfa in Fabry disease appears to be about $50 \%$. In subjects without Fabry disease, plasma $\mathrm{Gb}_{3}$ levels are not zero, but are reported to average between 3.5 (range, 2-11.2) ${ }^{32}$ and 5.6 (range, 3.6-7.5) (35 $^{35} \mathrm{~g} / \mathrm{mL}$ using sensitive high-performance liquid chromatography methods (these mean values convert to 3.7 and $5.9 \mu \mathrm{mol} / \mathrm{mL}$ based on a conversion of $\mu \mathrm{mol} / \mathrm{mL}=1.05 \mu \mathrm{g} / \mathrm{mL}^{33}$ ). In the pooled population of the present study, the mean plasma $\mathrm{Gb}_{3}$ level after 10 weeks of treatment with agalsidase alfa had fallen to $4.65 \pm$ $1.62 \mu \mathrm{mol} / \mathrm{mL}$, a value that could be considered within the normal range. Thus, the failure to detect a dose response in the present study likely reflects a near complete "normalization" of plasma $\mathrm{Gb}_{3}$ at all dosing regimens tested. The results of this study provide no information regarding the minimum effective dose of agalsidase alfa for fully reducing plasma $\mathrm{Gb}_{3}$. The lowest dose tested, $0.1 \mathrm{mg} / \mathrm{kg}$ weekly, is roughly equivalent to $0.2 \mathrm{mg} / \mathrm{kg}$ EOW and would be expected to have a similar effect on plasma $\mathrm{Gb}_{3}$ level. In fact, this equivalence was demonstrated in the study.

The cellular source of circulating $\mathrm{Gb}_{3}$ is not known, and it likely reflects excess $\mathrm{Gb}_{3}$ shed from a variety of organs and tissues, including the liver and vascular endothelial cells. Schiffmann et al..$^{23}$ reported that liver $\mathrm{Gb}_{3}$ content was reduced by $30 \%$ after a single infusion of agalsidase alfa and that the enzyme remained detectable within hepatic cells by immunohistochemistry 44 hours after dosing. Agalsidase alfa is primarily localized to vascular endothelial cells following intravenous administration in $\alpha$-gal A knockout mice, ${ }^{36}$ and long-term
ERT with agalsidase beta completely or nearly completely clears $\mathrm{Gb}_{3}$ from vascular endothelial cells. ${ }^{32,37}$ Thus, although it can be speculated that the reduction in plasma $\mathrm{Gb}_{3}$ is due to clearing of tissue stores from liver and vascular endothelial cells, further experiments would be necessary to demonstrate this putative link.

An interesting observation in this study was the persistence of the reduction in plasma $\mathrm{Gb}_{3}$ levels after cessation of ERT with agalsidase alfa. Four weeks after completing the study, the average plasma $\mathrm{Gb}_{3}$ level was $4.55 \pm 1.14 \mathrm{nmol} / \mathrm{mL}$, a value slightly less than that seen after 10 weeks of treatment. The persistence of this effect may reflect the prolonged time required for the reaccumulation of $\mathrm{Gb}_{3}$ in the primary cellular source of plasma $\mathrm{Gb}_{3}$.

Although changes in plasma and tissue or cellular $\mathrm{Gb}_{3}$ levels have been used to evaluate ERT in Fabry disease, $\mathrm{Gb}_{3}$ may not be a surrogate marker of Fabry disease. ${ }^{33} \mathrm{~A}$ recent study of the clinical and biochemical characteristics of 96 Dutch Fabry disease patients by Vedder and colleagues ${ }^{38}$ failed to demonstrate a correlation between plasma $\mathrm{Gb}_{3}$ level and the severity of the signs and symptoms of Fabry disease in patients with elevated plasma $\mathrm{Gb}_{3}$ (although statistically significant correlations were seen for left ventricular mass and Mainz Severity Score Index ${ }^{39}$ in male and female Fabry disease patients when patients without elevated plasma $\mathrm{Gb}_{3}$ levels were included in the analysis). In addition, no clinical studies have reported on the correlation between degree of reduction in plasma or cellular $\mathrm{Gb}_{3}$ level and clinical benefit. Until such studies are reported, changes in plasma $\mathrm{Gb}_{3}$ can only be used to evaluate enzyme activity after dosing and should not be used to infer efficacy.

\section{CONCLUSIONS}

Comparison of the dose response of agalsidase alfa on plasma $\mathrm{Gb}_{3}$ levels seen in the present study, combined with the observations reported for both agalsidase alfa and agalsidase beta in clinical studies, suggests that the approved dose of agalsidase alfa $(0.2 \mathrm{mg} / \mathrm{kg} \mathrm{EOW})$ is sufficient to produce maximal reduction of plasma $\mathrm{Gb}_{3}$ levels. However, because plasma $\mathrm{Gb}_{3}$ is not a validated surrogate of disease severity in Fabry disease, further clinical studies are needed to determine the optimum dosing regimen of ERT required to provide maximal improvement or stabilization of critical organ function and associated clinical benefits. This concept was recently exemplified by a study in a subpopulation of patients with advanced Fabry disease whose estimated glomerular filtration rate declined by $>5$ $\mathrm{mL} / \mathrm{min}$ per year on a long-term conventional dosing/frequency regimen of ERT. When the dosing frequency was switched to weekly, the rate of decline in loss of estimated glomerular filtration rate over the next 2 years was significantly slowed. ${ }^{40}$

\section{ACKNOWLEDGMENTS}

Shire HGT participated in the planning of this study and provided financial support for its conduct. Biochemical determination of plasma $\mathrm{Gb}_{3}$ levels and the incidence of antibodies 
were done at Shire HGT, as was all statistical analysis. This study was supported in part by the Intramural Program of the National Institute of Neurological Disorders and Stroke. Edward Weselcouch, $\mathrm{PhD}$. provided editorial assistance to the authors.

\section{References}

1. Brady RO, Gal AE, Bradley RM, Martensson E, et al. Enzymatic defect in Fabry's disease: ceramidetrihexosidase deficiency. N Engl J Med 1967;276:1163-1167.

2. Meikle PJ, Hopwood JJ, Clague AE, Carey WF. Prevalence of lysosomal storage disorders. JAMA 1999;281:249-254.

3. Spada M, Pagliardini S, Yasuda M, Tukel T, et al. High incidence of later-onset Fabry disease revealed by newborn screening. Am J Hum Genet 2006;79:31-40.

4. Brady RO, Schiffmann R. Clinical features of and recent advances in therapy for Fabry disease. JAMA 2000;284:2771-2775.

5. Pastores GM, Lien Y-HH. Biochemical and molecular genetic basis of Fabry disease. J Am Soc Nephrol 2002;13:S130-S133.

6. Ries M, Ramaswami U, Parini R, Lindblad B, et al. The early clinical phenotype of Fabry disease: a study on 35 European children and adolescents. Eur J Pediatr 2003; 162:767-772.

7. Ries M, Gupta S, Moore DF, Sachdev V, et al. Pediatric Fabry disease. Pediatrics 2005;115:e344-e355.

8. Ramaswami U, Whybra C, Parini R, Pintos-Morell G, et al. Clinical manifestations of Fabry disease in children: data from the Fabry Outcome Survey. Acta Paediatr 2006;95:86-92.

9. MacDermot KD, Holmes A, Miners AH. Anderson-Fabry disease: clinical manifestations and impact of disease in a cohort of 98 hemizygous males. J Med Genet 2001;38:750-760.

10. MacDermot KD, Holmes A, Miners AH. Anderson-Fabry disease: clinical manifestations and impact of disease in a cohort of 60 obligate carrier females. J Med Genet 2001;38:769-775.

11. Schiffmann R, Floeter MK, Dambrosia JM, Gupta S, et al. Enzyme replacement therapy improves peripheral nerve and sweat function in Fabry disease. Muscle Nerve 2003;28:703-710.

12. Ries M, Kim HJ, Zalewski CK, Mastroianni MA, et al. Neuropathic and cerebrovascular correlates of hearing loss in Fabry disease. Brain 2007;130:143-150.

13. Kampmann C, Baehner F, Ries M, Beck M. Cardiac involvement in Anderson-Fabry disease. J Am Soc Nephrol 2002;13:S147-S149.

14. Kampmann C, Baehner F, Whybra C, Martin C, et al. Cardiac manifestations of Anderson-Fabry disease in heterozygous females. J Am Coll Cardiol 2002;40:16681674.

15. Kampmann C, Wiethoff CM, Martin C, Wenzel A, et al. Electrocardiographic signs of hypertrophy in Fabry disease-associated hypertrophic cardiomyopathy. Acta Paediatr 2002;91:21-27.

16. Shah JS, Hughes DA, Sachdev B, Tome M, et al. Prevalence and clinical significance of cardiac arrhythmia in Anderson-Fabry disease. Am J Cardiol 2005;96:842-846.

17. Thadhani R, Wolf M, West ML, Tonelli M, et al. Patients with Fabry disease on dialysis in the United States. Kidney Int 2002;61:249-255.

18. Branton MH, Schiffmann R, Sabnis SG, Murray GJ, et al. Natural history of Fabry renal disease: influence of $\alpha$-galactosidase A activity and genetic mutations on clinical course. Medicine 2002;81:122-138.

19. Gold KF, Pastores GM, Botteman MF, Yeh JM, et al. Quality of life of patients with Fabry disease. Qual Life Res 2002;11:317-327.

20. Hoffmann B, Garcia de LA, Mehta A, Beck M, et al. Effects of enzyme replacement therapy on pain and health related quality of life in patients with Fabry disease: data from FOS (Fabry Outcome Survey). J Med Genet 2005;42:247-252.
21. Whybra C, Kampmann C, Willers I, Davies J, et al. Anderson-Fabry disease: clinical manifestations of disease in female heterozygotes. J Inherit Metab Dis 2001;24:715724.

22. Lee K, Jin X, Zhang K, Copertino L, et al. A biochemical and pharmacological comparison of enzyme replacement therapies for the glycolipid storage disorder Fabry disease. Glycobiology 2003;13:305-313.

23. Schiffmann R, Murray GJ, Treco D, Daniel P, et al. Infusion of $\alpha$-galactosidase A reduces tissue globotriaosylceramide storage in patients with Fabry disease. Proc Natl Acad Sci U S A 2000;97:365-370.

24. Schiffmann R, Kopp JB, Austin HAI, Sabnis S, et al. Enzyme replacement therapy in Fabry disease: a randomized controlled trial. JAMA 2001;285:2743-2749.

25. Banikazemi M, Bultas J, Waldek S, Wilcox WR, et al. Agalsidase-beta therapy for advanced Fabry disease: a randomized trial. Ann Intern Med 2007;146:77-86.

26. Altarescu G, Hill S, Wiggs E, Jeffries N, et al. The efficacy of enzyme replacement therapy in patients with chronic neuronopathic Gaucher's disease. J Pediatr 2001; 138:539-547.

27. Bishop DF, Desnick RJ. Affinity purification of $\alpha$-galactosidase A from human spleen, placenta, and plasma with elimination of pyrogen contamination. Properties of the purified splenic enzyme compared to other forms. J Biol Chem 1981;256: 1307-1316.

28. Schiffmann R, Brady RO. New prospects for the treatment of lysosomal storage diseases. Drugs 2002;62:733-742.

29. Eng CM, Banikazemi M, Gordon RE, Goldman M, et al. A phase 1/2 clinical trial of enzyme replacement in Fabry disease: pharmacokinetic, substrate clearance, and safety studies. Am J Hum Genet 2001;68:711-722.

30. Eng CM, Guffon N, Wilcox WR, Germain DP, et al. Safety and efficacy of recombinant human $\alpha$-galactosidase A replacement therapy in Fabry's disease. N Engl J Med 2001;345:9-16.

31. Desnick RJ. Enzyme replacement therapy for Fabry disease: lessons from two $\alpha$-galactosidase A orphan products and one FDA approval. Expert Opin Biol Ther 2004; 4:1167-1176.

32. Wilcox WR, Banikazemi M, Guffon N, Waldek S, et al. Long-term safety and efficacy of enzyme replacement therapy for Fabry disease. Am J Hum Genet 2004;75:65-74.

33. Bekri S, Lidove O, Jaussaud R, Knebelmann B, et al. The role of ceramide trihexoside (globotriaosylceramide) in the diagnosis and follow-up of the efficacy of treatment of Fabry disease: a review of the literature. Cardiovasc Hematol Agents Med Chem 2006;4:289-297.

34. Schiffmann R, Ries M, Timmons M, Flaherty JT, et al. Long-term therapy with agalsidase alfa for Fabry disease: safety and effects on renal function in a home infusion setting. Nephrol Dial Transplant 2006;21:345-354.

35. Young E, Mills K, Morris P, Vellodi A, et al. Is globotriaosylceramide a useful biomarker in Fabry disease? Acta Paediatr Suppl 2005;94:51-54.

36. Murray GJ, Anver MR, Kennedy MA, Quirk JM, et al. Cellular and tissue distribution of intravenously administered agalsidase alfa. Mol Genet Metab 2006;90:307312 .

37. Schiffmann R, Rapkiewicz A bu-Asab M, Ries M, et al. Pathological findings in a patient with Fabry disease who died after 2.5 years of enzyme replacement. Virchows Arch 2006;448:337-343.

38. Vedder AC, Linthorst GE, van Breemen MJ, Groener JE, et al. The Dutch Fabry cohort: diversity of clinical manifestations and $\mathrm{Gb}_{3}$ levels. J Inherit Metab Dis 2007; 30:68-78.

39. Whybra C, Kampmann C, Krummenauer F, Ries M, et al. The Mainz Severity Score Index: a new instrument for quantifying the Anderson-Fabry disease phenotype, and the response of patients to enzyme replacement therapy. Clin Genet 2004;65: 299-307.

40. Schiffmann R, Askari H, Timmons M, Robinson C, et al. Weekly enzyme replacement therapy may slow decline of renal function in patients with Fabry disease who are on long-term biweekly dosing. J Am Soc Nephrol 2007;18:1576-1583. 